\title{
DYNAMICS OF DUST PARTICLES RELEASED FROM OORT CLOUD COMETS AND THEIR CONTRIBUTION TO RADAR METEORS
}

\author{
David NesvornÝ ${ }^{1}$, David VokrouhlickÝ $\hat{y}^{1,2}$, Petr Pokorný ${ }^{1,2}$, and Diego Janches ${ }^{3}$ \\ ${ }^{1}$ Department of Space Studies, Southwest Research Institute, 1050 Walnut Street, Suite 300, Boulder, CO 80302, USA \\ ${ }^{2}$ Institute of Astronomy, Charles University, V Holešovičkách 2, CZ-18000, Prague 8, Czech Republic \\ ${ }^{3}$ Space Weather Laboratory, Code 674, GSFC/NASA, Greenbelt, MD 20771, USA \\ Received 2011 April 15; accepted 2011 September 5; published 2011 November 21
}

\begin{abstract}
The Oort Cloud Comets (OCCs), exemplified by the Great Comet of 1997 (Hale-Bopp), are occasional visitors from the heatless periphery of the solar system. Previous works hypothesized that a great majority of OCCs must physically disrupt after one or two passages through the inner solar system, where strong thermal gradients can cause phase transitions or volatile pressure buildup. Here we study the fate of small debris particles produced by OCC disruptions to determine whether the imprints of a hypothetical population of OCC meteoroids can be found in the existing meteor radar data. We find that OCC particles with diameters $D \lesssim 10 \mu \mathrm{m}$ are blown out from the solar system by radiation pressure, while those with $D \gtrsim 1 \mathrm{~mm}$ have a very low Earth-impact probability. The intermediate particle sizes, $D \sim 100 \mu \mathrm{m}$, represent a sweet spot. About $1 \%$ of these particles orbitally evolve by Poynting-Robertson drag to reach orbits with semimajor axis $a \sim 1 \mathrm{AU}$. They are expected to produce meteors with radiants near the apex of Earth's orbital motion. We find that the model distributions of their impact speeds and orbits provide a good match to radar observations of apex meteors, except for the eccentricity distribution, which is more skewed toward $e \sim 1$ in our model. Finally, we propose an explanation for the long-standing problem in meteor science related to the relative strength of apex and helion/antihelion sources. As we show in detail, the observed trend, with the apex meteors being more prominent in observations of highly sensitive radars, can be related to orbital dynamics of particles released on the long-period orbits.
\end{abstract}

Key words: meteorites, meteors, meteoroids - zodiacal dust

\section{INTRODUCTION}

The Oort Cloud is a roughly spherical cloud of comets (Oort 1950), which surrounds the solar system and extends to heliocentric distances larger than 100,000 AU. The Oort Cloud is currently feeding comets into the inner solar system at a rate of about 12 comets with $q<3 \mathrm{AU} \mathrm{yr}^{-1}$ with an active comet absolute magnitude $H_{10}<10.9$ (Wiegert \& Tremaine 1999; $q$ is the perihelion distance and $H_{10}$ is a distance-independent measure of the active comet brightness that includes the coma). The Oort Cloud Comets (hereafter OCCs) can be divided into two populations based on their dynamical histories: (1) dynamically new OCCs, which are on their first passage through the inner solar system and typically have $a \gtrsim 10,000 \mathrm{AU}$ and (2) returning OCCs which have previously passed through the inner solar system and typically have $a<10,000 \mathrm{AU} .{ }^{4}$

The dynamical models of the orbital evolution of new OCCs into returning OCCs predict many times more returning comets than are observed (Wiegert \& Tremaine 1999). This is the socalled fading problem, which is thought to be related to the physical evolution of OCCs. To resolve this problem, Levison et al. (2002, hereafter L02) proposed that OCCs must physically disrupt as they evolve inward from the Oort Cloud. Specifically, L02 estimated that, when an OCC becomes inactive, it has only $\sim 1 \%$ chance of becoming dormant, and $\sim 99 \%$ chance of being disrupted. If more OCCs would become dormant, L02 argued, the modern surveys of near-Earth objects would discover a greater number of dormant OCCs passing through perihelion each year than they do. Strong thermal gradients, phase transitions, and volatile pressure buildup experienced by

\footnotetext{
4 See Dybczyński (2001) for an alternative definition.
}

OCCs during their approaches to the Sun are thought to be responsible for disruptions.

If these results are correct, the disrupted OCCs must be a prodigious source of dust particles and larger fragments that may further disintegrate. In particular, the dust production rate from OCC disruptions should be vastly larger than that of active OCCs. On the other hand, the smallest dust particles produced in these disruption events may be lost from the solar system due to the effects of radiation pressure, while the large fragments should be dispersed over enormous radial distances. It is therefore not clear whether the disrupted OCCs can supply a significant amount of material to the inner zodiacal cloud, and whether they could represent a significant source of interplanetary dust particles accreted by the Earth.

Given their large speeds relative to the Earth, the OCC particles plunging into the upper atmosphere could produce meteor phenomena and be detected by optical and radar meteor surveys (e.g., see Steel 1996 for a review). Here we consider the radar meteors. The modern meteor radar surveys produced vast data sets including millions of high-quality orbits covering both the northern and southern hemispheres (e.g., Jones \& Brown 1993; Taylor \& Elford 1998; Galligan \& Baggaley 2004, 2005; Janches et al. 2003; Janches \& Chau 2005; Chau et al. 2007; Campbell-Brown 2008). Moreover, the sensitivity of meteor observations broke new grounds with routine measurements of meteor echoes using HPLA $^{5}$ radars such as the Arecibo (AO) radar, which is capable of detecting $\sim 50 \mu \mathrm{m}$ particles down to $\sim 20 \mathrm{~km} \mathrm{~s}^{-1}$ (e.g., Janches et al. 2003, 2006; Fentzke et al. 2009). It is natural to ask whether some of these observations can be linked to the particle populations from disrupted OCCs.

\footnotetext{
5 High Power and Large Aperture.
} 
Meteors are produced by small interplanetary particles, also known as the meteoroids, that interact with air molecules upon atmospheric entry. Based on meteor data, the meteoroids can be divided into two groups: sporadic meteoroids and meteoroid streams. The meteoroid streams are prominent concentrations of particles with similar orbits (Whipple \& Gossner 1949; Whipple 1951). They are thought to be produced by particles released by active and recently ( $<$ few thousand years ago) disrupted comets (e.g., Jenniskens 2008). Sporadic meteoroids are those particles that have evolved significantly from their parent body so that they are no longer easily linked to that parent, or to other meteoroids from the same parent. Notably, the time-integrated flux of meteors at Earth is dominated by about a factor of $\sim 10$ by sporadics (Jones \& Brown 1993).

The radiant distribution of sporadic meteors shows several concentrations on the sky, known as the helion/antihelion, north/south apex, and north/south toroidal sources (e.g., Younger et al. 2009, and references therein). Wiegert et al. (2009, hereafter W09) developed a dynamical model to explain these concentrations. Their main results concern the prominent helion/antihelion sources for which the particles released by Jupiter-family comets (JFCs) such as $2 \mathrm{P} /$ Encke provide the best match, in agreement with previous studies (e.g., Jones et al. 2001). As for the north/south apex source, W09 pointed out the potential importance of retrograde Halley-type comets (HTCs) such as 55P/Tempel-Tuttle (or an orbitally similar lost comet). The case for the retrograde HTC particles is compelling, because three prominent retrograde HTCs, namely, 1P/Halley, 55P/Tempel-Tuttle, and 109P/Swift-Tuttle, all have associated streams, known as Orionids/ $\eta$ Aquarids, Leonids, and Perseids, respectively. The sporadic meteoroids with the north/south apex radiants can thus plausibly be a dynamically old component of HTC particles.

Here we consider the possibility that at least some part of the meteoroid complex is produced by disrupting OCCs (L02; see also Jones et al. 2001). We study the effects of radiation pressure on particles released from the highly eccentric OCC orbits, and their dynamical evolution under gravitational perturbations from planets and Poynting-Robertson (P-R) drag (see Section 2 for our model). We show that a significant contribution of OCC particles to the inner zodiacal cloud and meteor record is somewhat problematic, because most small OCC particles are blown out from the solar system by radiation pressure, while most large ones get scattered by planets and never make it into the inner solar system (Section 3). Still, we find that there is a sweet spot at particle sizes $\sim 100-300 \mu \mathrm{m}$. Our modeling work shows that the orbits and impact speeds of these intermediatesize OCC particles can match those derived from the meteor radar data for apex meteoroids. Furthermore, we find that the preponderance of fast apex meteors in HPLA radar observations (e.g., AO, ALTAIR, Jicamarca) can be linked to the competing effects of P-R drag and Jupiter perturbations, which act as a size filter on populations of the long-period meteoroids (Section 4).

\section{MODEL}

We studied the following sequence of events: (1) particles of different sizes were released from OCCs (Section 2.1), (2) their orbits evolved under the influence of gravitational and radiation forces (Section 2.2), (3) some particles were thermally or collisionally destroyed (Section 2.3), and (4) a small fraction of the initial particle population was accreted by Earth, producing meteors (Section 2.4). We describe our model for (1)-(4) below.

\subsection{Initial Orbits}

According to Francis (2005), OCCs have $d N(q) \propto(1+$ $\sqrt{q}) d q$ for $q<2$ AU. For $q>2$ AU, Francis' result predicts $d N(q)$ being flat or declining, while we would expect the perihelion distribution to increase with $q$. It probably just shows that the distribution is not well constrained for $q>2 \mathrm{AU}$. We used $d N(q) \propto 2.41(q / 2)^{\gamma} d q$ for $q>2 \mathrm{AU}$, with $0 \leqslant \gamma \leqslant 1$. The initial values of $q$ in our numerical integrations were set to be uniformly random between 0 and $5 \mathrm{AU}$, because particles starting with $q>5$ AU do not reach 1 AU (see Section 3.1), where they could contribute to the Earth-impact record. The results for different $d N(q)$ were obtained by assigning the appropriate weight to particles starting with different $q$ s (i.e., instead of 1 we counted each particle as $d N(q) / d q)$.

Upon its release from a larger object, a small particle will feel the effects of radiation pressure. These effects can be best described by replacing the mass of the Sun, $m_{\odot}$, by $m_{\odot}(1-\beta)$, with $\beta$ given by

$$
\beta=5.7 \times 10^{-5} \frac{Q_{\mathrm{pr}}}{\rho s},
$$

where radius $s$ and density $\rho$ of the particle are in cgs units. Pressure coefficient $Q_{\mathrm{pr}}$ can be determined using the Mie theory (Burns et al. 1979). We set $Q_{\mathrm{pr}}=1$, which corresponds to the geometrical optics limit, where $s$ is much larger than the incident-light wavelength. We used particles with $D=2 s=10$, $30,100,300,1000 \mu \mathrm{m}$, which should cover the interesting range of sizes, and $\rho=2.0 \mathrm{~g} \mathrm{~cm}^{-3}$.

For large eccentricity $e$ of the parent object and/or for large $\beta$, the released particle may become unbound and escape to interstellar space. To stay bound, the heliocentric distance, $R$, of the released particle must fulfill the following condition (e.g., Kresak 1976; Liou et al. 1999):

$$
R>R^{*}=2 \beta a .
$$

This condition shows that all particles with $\beta$ released at the orbit's perihelion will be removed, if $2 \beta>1-e$. The new OCCs have $1-e \lesssim 10^{-4}$. It follows that particles produced by a new OCC near its perihelion will become unbound for sizes up to $D \sim 1 \mathrm{~cm}$. The usual near-perihelion activity of OCCs therefore cannot be a major source of small dust particles in the inner solar system.

Motivated by the L02 results, we now consider OCCs disruptions. Interestingly, observations of the disruption events of comets show that there does not seem to be any correlation between the time of disruption and the orbital phase of the parent object. Many comets were seen to disrupt (or suffer outburst/splitting events) at large heliocentric distances. For example, $174 \mathrm{P} /$ Echeclus showed an outburst with $R \approx 13 \mathrm{AU}$, more than 6 AU beyond its perihelion distance (Choi et al. 2006). It may therefore be possible that OCCs could disrupt at relatively large $R$ and produce particles that, according to Equation (2), will stay on bound orbits.

We release particles with $R>R^{*}$ in our model. For example, a $D=100 \mu \mathrm{m}$ particle with $\rho=2.0 \mathrm{~g} \mathrm{~cm}^{-3}$ ejected from the parent comet with $a=10^{3}$ AU will have $\beta \simeq 0.006$ and $R^{*}=12 \mathrm{AU}$. We thus release these particles with $R>12 \mathrm{AU}$. In addition, we only study particles ejected from orbits similar to those of the returning OCCs with $a \sim 10^{3}$ AU. We do not consider orbits with $a \gtrsim 10^{4} \mathrm{AU}$, corresponding to the 
Oort spike, ${ }^{6}$ because we believe it unlikely that disruptions could happen at the very large heliocentric distance implied by Equation (2) for $a \gtrsim 10^{4}$ AU. For example, a $D=300 \mu \mathrm{m}$ particle with $\rho=2.0 \mathrm{~g} \mathrm{~cm}^{-3}$, released from a parent orbit with $a=10^{4}$ AU, would become unbound, unless $R>36 \mathrm{AU}$.

The particle populations studied here have bound initial orbits. They represent only a fraction of all particles released from OCCs. This fraction, denoted $f_{0}$ in Section 4 , is difficult to estimate, because we do not have a detailed understanding of the processes, and their dependence on $R$, that govern comet disruptions. We will return to this issue in Section 4. The initial distribution of orbital inclination vectors was set to be isotropic. To simplify things, we neglected the ejection velocities of dust particles from their parent objects (see Jones et al. 2001) and assumed that they will initially follow the parent comet's orbit modified by radiation pressure.

For reference, we also followed meteoroids from $1 \mathrm{P} / \mathrm{Halley}$, 2P/Encke, and 55P/Tempel-Tuttle. These comets were suggested to be important sources of the sporadic meteors by W09. The comet's orbits were obtained from the JPL Horizons site. Particles of different sizes were released from the parent orbits and tracked into future. We applied the same procedures/criteria to them that we used for the OCC particles.

\subsection{Orbit Integration}

The orbits of small particles in the interplanetary space are subject to gravitational perturbations of planets and radiation forces (Robertson 1937; Burns et al. 1979). The acceleration $\vec{F}$ due to radiation forces is

$$
\vec{F}=\beta G \frac{m_{\odot}}{R^{2}}\left[\left(1-\frac{\dot{R}}{c}\right) \frac{\vec{R}}{R}-\frac{\vec{V}}{c}\right],
$$

where $\vec{R}$ is the heliocentric position vector of particle, $\vec{V}$ is its velocity, $G$ is the gravitational constant, $m_{\odot}$ is the mass of the Sun, $c$ is the speed of light, and $\dot{R}=d R / d t$. The acceleration Equation (3) consists of the radiation pressure and the velocitydependent P-R term. Parameter $\beta$ is related to the radiation pressure coefficient $Q_{\mathrm{pr}}$ by Equation (1).

The particle orbits were numerically integrated with the swift_rmvs3 code (Levison \& Duncan 1994), which is an efficient implementation of the Wisdom-Holman map (Wisdom \& Holman 1991) and which, in addition, can deal with close encounters between particles and planets. The radiation pressure and drag forces were inserted into the Keplerian and kick parts of the integrator, respectively. The change to the Keplerian part was trivially done by substituting $m_{\odot}$ by $m_{\odot}(1-\beta)$. The swift_rmvs3 integrator is stable even for near-parabolic orbits, and thus well suited for the integrations that we carried out here.

The code tracks the orbital evolution of a particle that revolves around the Sun and is subject to the gravitational perturbations of seven planets (Venus to Neptune; the mass of Mercury was

\footnotetext{
6 The semimajor axis values of most OCCs are $10^{4} \lesssim a \lesssim 5 \times 10^{4}$ AU, which is known as the Oort spike (e.g., Wiegert \& Tremaine 1999). Comets in the spike are mostly dynamically new comets, on their first passage into the inner planetary system from the Oort Cloud. A comet that passes through the planetary system receives a gravitational kick from the planets. The typical energy kick, $\Delta x$, depends strongly on the perihelion distance of the comet's orbit. According to Wiegert \& Tremaine (1999), $\Delta x \sim 10^{-3} \mathrm{AU}^{-1}$ for $q \lesssim 6$ $\mathrm{AU}$, while comets in the Oort spike have $x=1 / a \lesssim 10^{-4} \mathrm{AU}^{-1}$. Depending on the sign of the kick, they will either leave the planetary system on unbound orbit, never to return, or be thrown onto a more tightly bound orbit with $a \lesssim 10^{3} \mathrm{AU}$.
}

added to the Sun) until the particle impacts a planet, is ejected from the solar system or evolves to within $0.05 \mathrm{AU}$ from the Sun. We removed particles that evolved to $R<0.05$ AU because the orbital period for $R<0.05 \mathrm{AU}$ was not properly resolved by our 1 day integration time step.

Several thousand particles were followed for each $D$. Their orbital elements were defined with respect to the barycenter of the solar system. The barycentric elements are similar to the heliocentric elements for $R<5 \mathrm{AU}$, but differ for large $R$, where the Sun's orbital speed about the barycenter of the solar system is not negligible relative to the orbital speed of a particle.

All orbits were followed from the present epoch into the future. Each particle's orbital elements were stored at $10^{3} \mathrm{yr}$ intervals. We used the output to construct a steady state distribution of OCC particles in the inner solar system. This approach differs from that of Nesvorný et al. (2006) and W09, who started particles at many different past epochs and used these integrations to determine the present distribution of particles. The two distributions are expected to be slightly different, expressing mainly the difference between the present configuration of planets, with each planet having a specific secular phase, and the time-averaged system, where all phases are mixed. Since this difference is small, however, we can use the steady state distribution, which is easier to obtain, as a reasonable approximation.

\subsection{Physical Effects}

Solar system micrometeoroids can be destroyed by collisions with other particles and by solar heating, that can lead to sublimation and vaporization of minerals. Here we describe how we parameterize these processes in our model.

\subsubsection{Thermal Destruction}

Thermal alteration of grains in the interplanetary grains is a complex process. The OCC particles evolving into the inner solar system will first lose their volatile ices, which will rapidly sublimate once the grains are heated to a critical temperature. We do not model the volatile loss in detail. Instead, we crudely assume that the grains have lost $\sim 50 \%$ of their mass/volume when reaching $R \lesssim 5$ AU. We do not include the orbital effects of mass loss in orbital modeling because it should produce only a relatively small perturbation on orbits for large particles that we consider here. The remaining grains will be primarily composed from amorphous silicates and will survive down to very small $R$.

According to Duschl et al. (1996), silicates are thermally altered at temperatures $T \sim 900-1600 \mathrm{~K}$, and start to vaporize for $T>1600 \mathrm{~K}$. As an example of thermal alteration, Kasuga et al. (2006; see also Čapek \& Borovička 2009) studied the thermal desorption of alkali minerals and concluded that micrometeoroids should show evidence of thermal desorption of metals, $\mathrm{Na}$ in particular, for $q<0.1$ AU. Following Moro-Martín \& Malhotra (2002), Kessler-Silacci et al. (2007), and others, we adopt a simple criterion for the silicate grain destruction. We will assume that they are destroyed when the grain temperature reaches $T=1500 \mathrm{~K}$.

The temperature of a small, fast spinning grain in interplanetary space is set by an equilibrium between the absorbed and re-radiated energy fluxes. While the absorbed flux is a simple function of the particle's size, albedo and heliocentric distance, the re-radiated flux depends on the particle's emissivity, which in turn is a function of the particle's size, shape, and material 
properties. Using the optical constants of amorphous pyroxene of approximately cosmic composition (Henning \& Mutschke 1997), we find that a dark $D \gtrsim 100 \mu \mathrm{m}$ grain at $R$ has the equilibrium temperature within $10 \mathrm{~K}$ of a blackbody, $T(R) \simeq 275 / \sqrt{R} \mathrm{~K}$. According to our simple destruction criterion, $T(R)>1500 \mathrm{~K}$, the silicate grains should thus be removed when reaching $R \lesssim 0.03$ AU. On the other hand, the smallest particles considered in this work, $D=10 \mu \mathrm{m}$, will reach $T(R)=1500 \mathrm{~K}$ for $R \simeq 0.05 \mathrm{AU}$. Thus, we opted for using a very simple (and conservative) criterion where particles of all sizes were destroyed, and not considered for statistics, if they ever reached $R \leqslant 0.05 \mathrm{AU}$. Note that, by design, this limit is the same as the one imposed by the integration time step (Section 2.2).

\subsubsection{Disruptive Collisions}

The collisional lifetime of meteoroids, $\tau_{\text {coll }}$, was taken from Grün et al. (1985, hereafter G85). It was assumed to be a function of particle mass, $m$, and orbital parameters, mainly $a$ and $e$. We neglected the effect of orbital inclination on $\tau_{\text {coll }}$, because the results discussed in Steel \& Elford (1986) suggest that the inclinations should affect $\tau_{\text {coll }}$ only up to a factor of $\sim 2-5$, which is not overly significant in the context of our work. We assumed that the mass and orbital dependencies of $\tau_{\text {coll }}$ can be decoupled, so that

$$
\tau_{\text {coll }}(m, a, e)=\Phi(m) \Psi(a, e),
$$

where $\Phi(m)$ and $\Psi(a, e)$ are discussed below.

As for $\Phi(m)$, we used the G85 model based on measurements of various spacecraft and Earth-based detectors. We found that the model can be approximated by a simple empirical fit. Specifically, between $10^{-6.4} \mathrm{~g}$ and $10^{1.2} \mathrm{~g}$, we adopted the quadratic function

$$
\log \Phi(m)=c_{2}(\log m)^{2}+c_{1}(\log m)+c_{0},
$$

with $\left(c_{0}, c_{1}, c_{2}\right)=(4.021,0.300,0.083)$, where the values of $c_{0}$, $c_{1}$, and $c_{2}$ were set to fit the G85 collision lifetime for circular orbits at the reference distance $R_{0}=1 \mathrm{AU}$. A linear relation between $\log \Phi(m)$ and $\log m$ was used outside the quoted mass range to approximate the G85 model down to $m=10^{-8} \mathrm{~g}$. $\Phi(\mathrm{m})$ has a minimum for $m \simeq 0.01 \mathrm{~g}$, corresponding to $s \simeq 1 \mathrm{~mm}$ for $\rho=2 \mathrm{~g} \mathrm{~cm}^{-3}$ (Figure 1). The collisional lifetime of $\sim 1 \mathrm{~mm}$ particles in the G85 model is very short, roughly $5000 \mathrm{yr}$ at $1 \mathrm{AU}$.

$\Psi(a, e)$ is assumed to drop as a power law with $R$. From Equation (18) in G85 we have

$$
\Psi=\left(\frac{R}{R_{0}}\right)^{\alpha} \frac{v(R)}{v_{\text {circ }}(R)},
$$

where $v(R)$ and $v_{\text {circ }}(R)$ are the particle and circular speeds at $R$, respectively, and $\alpha \simeq 1.8$. The velocity-dependent factor provides an appropriate scaling of $\tau_{\text {coll }}$ for eccentric orbits.

Averaging Equation (6) over a Keplerian orbit with semimajor axis $a$ and eccentricity $e$, we obtain

$$
\Psi(a, e)=\left(\frac{a}{R_{0}}\right)^{\alpha} \mathcal{J}(e),
$$

where

$$
\mathcal{J}(e)=\frac{1}{\pi} \int_{0}^{\pi} d u \frac{(1-e \cos u)^{1+\alpha}}{\sqrt{1+e \cos u}}
$$

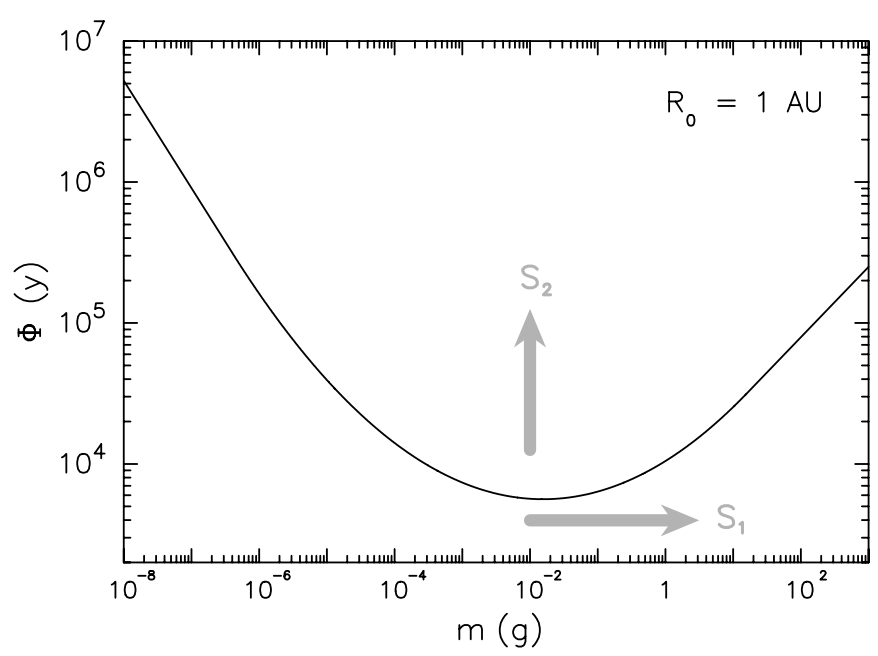

Figure 1. Mass dependence, $\Phi(m)$, of the adopted model for the collisional lifetime of particles. The plot shows $\Phi(m)$ for a particle on the circular orbit at the reference heliocentric distance $R_{0}=1 \mathrm{AU}$. Two parameters of the collisional model, $S_{1}$ and $S_{2}$, were used to test the sensitivity of our results to modifications of $\Phi(m)$.

can be written as a series in $e^{2}$ with good convergence. Note that $\mathcal{J} \simeq 1$ for $e \simeq 0$, as required in Equation (7), but can become $\gg 1$ for very eccentric orbits.

The G85 model was calibrated to match the impact fluxes of particles as measured prior to 1985. The more recent measurements indicate lower fluxes (e.g., Dikarev et al. 2005; Drolshagen et al. 2008). Also, to estimate $\tau_{\text {coll }}$, assumptions needed to be made in G85 about the strength of particles, and their impact speeds. As a result, $\tau_{\text {coll }}$ proposed by G85 may have a significant uncertainty. To test different possibilities, we introduced two free parameters in our model, $S_{1}$ and $S_{2}$, that were used to shift the $\Phi(m)$ function in $\log m$ and $\log \Phi$, respectively (as indicated by arrows in Figure 1). For example, the positive $S_{2}$ values increase $\tau_{\text {coll }}$ relative to the standard G85 model, as expected, for example, if particles were stronger than assumed in G85, or if the fluxes were lower.

Collisional disruption of particles was taken into account during processing the output from the numerical integration described in Section 2.2. To account for the stochastic nature of breakups, we determined the breakup probability $p_{\text {coll }}=$ $1-\exp \left(-h / \tau_{\text {coll }}\right)$, where $h=1000 \mathrm{yr}$ is the output interval and $\tau_{\text {coll }}$ was computed individually for each particle's orbit. The code then generated a random number $0 \leqslant x \leqslant 1$, and eliminated the particle if $x<p_{\text {coll }}$.

We caution that our procedure does not take into account the small debris fragments that are generated by disruptions of larger particles. Instead, all fragments are removed from the system. This is an important approximation, whose validity needs to be tested in the future.

\subsection{Model for Meteor Radar Observations}

We used the Öpik theory (Öpik 1951) to estimate the expected terrestrial accretion rate of OCC particles in our model. Wetherill (1967), and later Greenberg (1982), improved the theory by extending it more rigorously to the case of two eccentric orbits. Here we used the Fortran program written by W. F. Bottke (see, e.g., Bottke et al. 1994), which employs the Greenberg formalism.

We modified the code to compute the radiants of the impacting bodies. The radiants were expressed in the coordinate system, 
where longitude $l$ was measured from Earth's apex in the counterclockwise direction along Earth's orbit and latitude $b$ was measured relative to Earth's orbital plane. Note that our definition of longitude is different from the one more commonly used for radar meteors, where the longitude is measured from the helion direction. The radiants were calculated before the effects of gravitational focusing were applied.

The longitude and latitude values of radiants were binned into $1 \mathrm{deg}^{2}$ area segments. For each radiant bin, the code gives information about the distribution of geocentric impact speeds, $v_{g}$, and heliocentric orbits prior to the impact, as defined by $a, e$, and $i$. Here, $v_{g}$ is defined as $v_{g}=\left(v_{\infty}^{2}+v_{\text {esc }}^{2}\right)^{1 / 2}$, where $v_{\infty}$ is the relative velocity "in infinity" and $v_{\text {esc }}$ is the escape speed from Earth's surface. The orbital elements, on the other hand, are the orbital elements that the particle would have in the absence of the gravitational focusing by the Earth. The radiant distribution, $v_{g}, a, e$, and $i$ will be compared to meteor radar observations in Section 3.3.

To compare our model with observations, we need to include the meteor radar detection efficiency. This is a difficult problem because the meteor phenomenon itself and radar detection of it involve complex physics. For example, the specular meteor radars (SMRs), such as the Canadian Meteor Orbit Radar (CMOR; Campbell-Brown 2008) and Advanced Meteor Orbit Radar (AMOR; Galligan \& Baggaley 2004, 2005), detect the specular reflection of the meteor trail (the plasma formed by the meteoroid's passage). The meteoroid velocities are then derived from the detection of the Fresnel diffraction patterns of the developing trail, or are determined by measuring the time of flight between stations.

The detection efficiency of a meteor should mainly be a function of the particle size and speed, but it also depends on a number of other parameters discussed, for example, in Janches et al. (2008). Following W09, we opt for a simple parameterization of radar sensitivity function, where the detection is represented by an ionization function

$$
I\left(m, v_{g}\right)=\frac{m}{10^{-4} \mathrm{~g}}\left(\frac{v_{g}}{30 \mathrm{~km} \mathrm{~s}^{-1}}\right)^{3.5} .
$$

All meteors with $I\left(m, v_{g}\right) \geqslant I^{*}$ are assumed to be detected in our model, while all meteors with $I\left(m, v_{g}\right)<I^{*}$ are not detected (see Fentzke et al. 2009 for a similar method applied to head echo radars). The ionization cutoff $I^{*}$ is different for different SMRs. For example, $I^{*} \sim 1$ for CMOR (CampbellBrown 2008) and $I^{*} \sim 0.001-0.01$ for AMOR (Galligan \& Baggaley 2004, 2005). For reference, an OCC particle with $v_{g}=60 \mathrm{~km} \mathrm{~s}^{-1}$ and $m=10^{-5} \mathrm{~g}$, corresponding to $s \sim 100 \mu \mathrm{m}$, will have $I\left(m, v_{g}\right) \simeq 0.1$, i.e., a value intermediate between the two thresholds. These meteoroids would thus be detected by AMOR, according to our definition, but not by CMOR. We will discuss these issues in more detail in Section 3.3.

\section{RESULTS}

\subsection{Orbital Evolution of OCC Particles}

Wyatt \& Whipple (1950) identified the following constant of motion of P-R drag

$$
C=a \frac{\left(1-e^{2}\right)}{e^{4 / 5}},
$$

where $a$ and $e$ are the particle's semimajor axis and eccentricity (see also Breiter \& Jackson 1998). This constant is independent
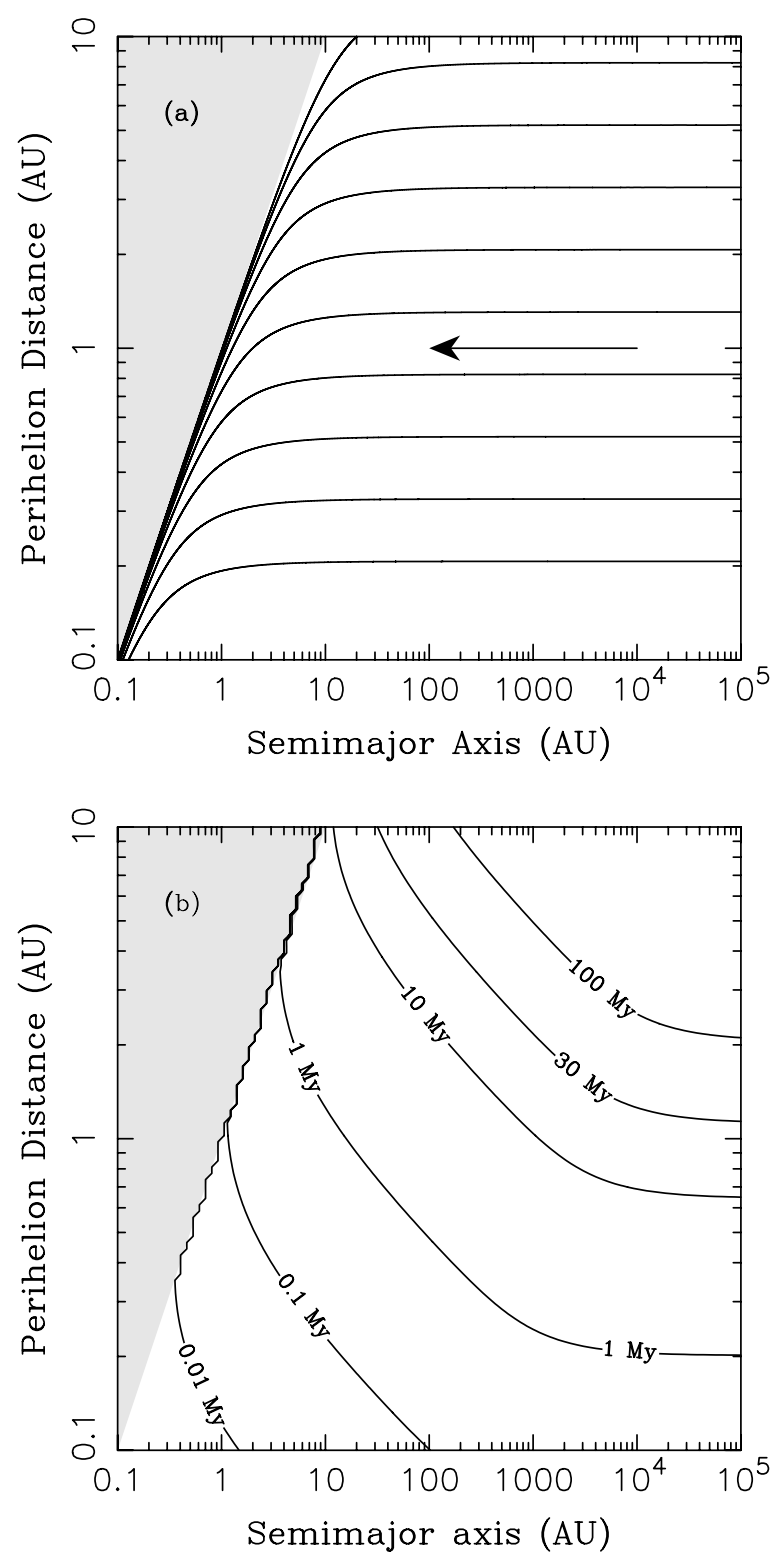

Figure 2. Effects of P-R drag on the highly eccentric orbits of OCC particles. (a) Evolution tracks of particles evolving by P-R drag in semimajor axis and perihelion distance. Particles with $e \simeq 1$ evolve from right to left along the lines of constant perihelion distance. The shaded area is inaccessible to orbits. (b) Evolution timescale for particles with $D=100 \mu \mathrm{m}$ and $\rho=2 \mathrm{~g} \mathrm{~cm}^{-3}$. Contours show the time of fall, $\tau_{\text {fall }}$, from the initial orbit with $a$ and $q$ to the Sun. According to Equation (11), $\tau_{\text {fall }}$ scales linearly with $D$ (and $\rho$ ), so that, for example, the $1 \mathrm{Myr}$ contour for $D=100 \mu \mathrm{m}$ is the $10 \mathrm{Myr}$ contour for $D=1 \mathrm{~mm}$.

of the particle properties such as its size. The orbit path of any particle in $a$ and $e$ can thus be obtained by calculating $C$ for the initial orbit, and requiring that Equation (10) holds at all times (Figure 2(a)). For $e \sim 1$, the orbit trajectories follow the lines of constant $q$, because $C \simeq 2 q$ for $e \simeq 1$. The semimajor axis of orbits shrinks until reaching a value only several times larger than $q$. At that point, a more familiar form of P-R drag takes place with both $a$ and $q$ converging to zero.

The timescale of orbital evolution is as follows. For an initial orbit with $a, e$, and $q=a(1-e)$, the total time of fall to the Sun is (Wyatt \& Whipple 1950)

$$
\tau_{\text {fall }}=20 \operatorname{Myr}\left(\frac{s}{1 \mathrm{~cm}}\right)\left(\frac{\rho}{2 \mathrm{~g} \mathrm{~cm}^{-3}}\right)\left(\frac{q}{1 \mathrm{AU}}\right)^{2} f(e),
$$



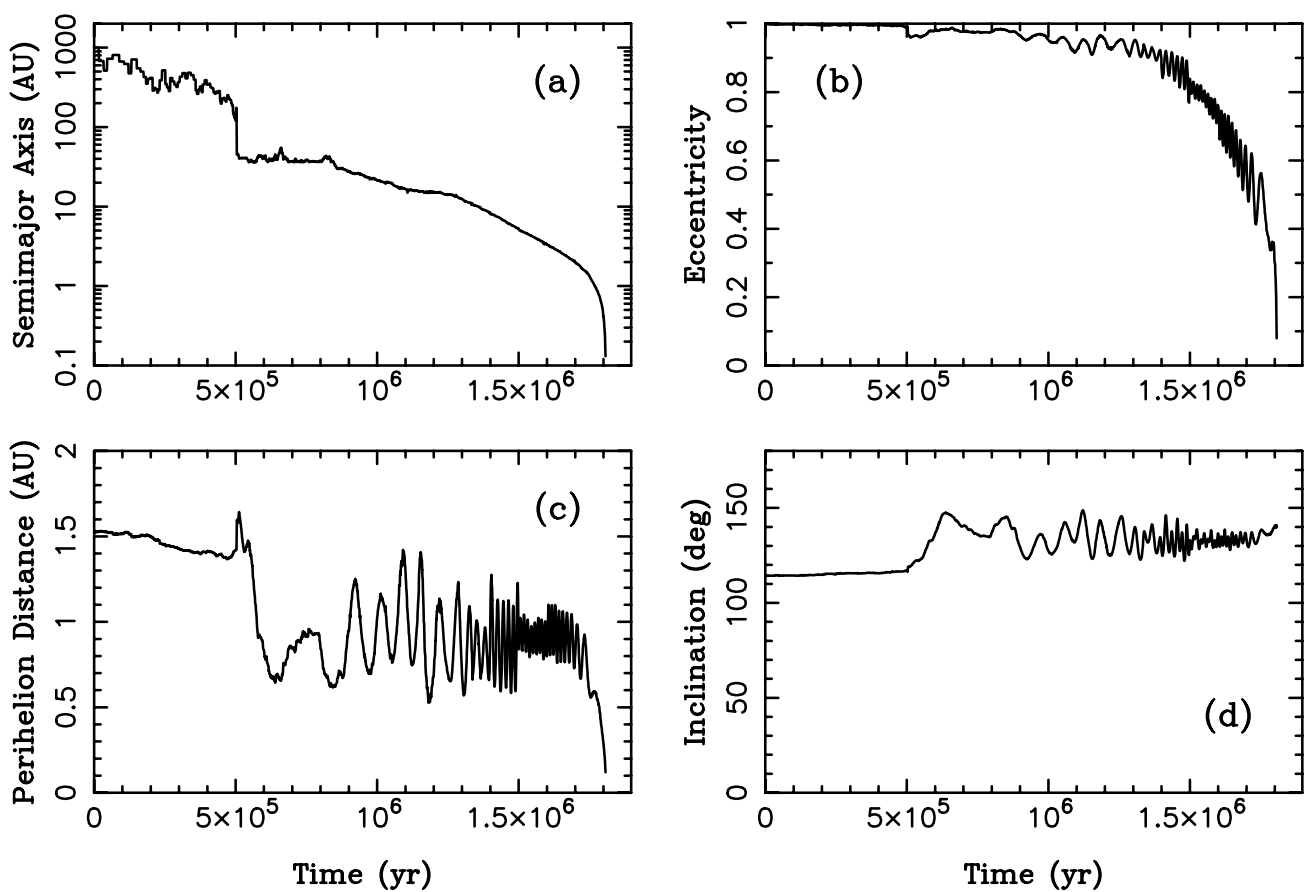

Figure 3. Orbit history of a particle with $D=100 \mu \mathrm{m}$, initial $a=10^{3} \mathrm{AU}$, and $q=1.53 \mathrm{AU}$. After $1.7 \mathrm{Myr}$, the orbit decouples from Jupiter and moves to $a \sim 1$ AU. The particle ends up having $a<0.1 \mathrm{AU}$, and sublimates upon reaching $R<0.05 \mathrm{AU}$.

where

$$
f(e)=1.13 \frac{(1+e)^{2}}{e^{8 / 5}} \int_{0}^{e} d \eta \frac{\eta^{3 / 5}}{\left(1-\eta^{2}\right)^{3 / 2}} .
$$

Figure 2(b) shows $\tau_{\text {fall }}$ for orbits relevant to OCC particles.

Interestingly, the timescale for large $a$ can be relatively short if $q$ is small. For example, an OCC particle with $D=100 \mu \mathrm{m}$, $a=10^{4} \mathrm{AU}$, and $q=1 \mathrm{AU}$ takes about $20 \mathrm{Myr}$ to fall to the Sun. This is only about 20 orbital periods for $a=10^{4}$ AU. According to Equation (11), $\tau_{\text {fall }}$ scales linearly with particle size. Thus, a $D=10 \mu \mathrm{m}$ particle with the same initial orbit has $\tau_{\text {fall }}=2$ Myr.

One important aspect that cannot be captured by the analytical results discussed above is the effect of planetary perturbations on drifting orbits of OCC particles. To evaluate this effect, as described in Section 2.2, we numerically integrated the orbits of OCC particles as they evolve from large $a$ and interact with the planets.

Figure 3 shows the orbital history of a particle, whose orbit evolved all the way down into the inner solar system. Initially, the particle's semimajor axis underwent a random walk caused by indirect planetary perturbations, mainly from Jupiter, during each perihelion passage (Wiegert \& Tremaine 1999). Then, at time $t \simeq 5 \times 10^{5} \mathrm{yr}$, a single perihelion passage produced a significant drop of $a$ from 150 to $40 \mathrm{AU}$, where the particle started to interact with the exterior mean motion resonances with Neptune. The following evolution was mainly controlled by P-R drag. Eventually, the orbit decoupled from Jupiter, reached $a \sim 1 \mathrm{AU}$, and kept shrinking further toward the Sun, where the particle was thermally destroyed. The large oscillations of $q$ for $0.9<t<1.7 \mathrm{Myr}$, correlated with those in $i$, were produced by Kozai dynamics (e.g., Kozai 1962).

The orbit history shown in Figure 3 is typical for an OCC particle that is able to make it into the inner solar system. These particles, however, represent a relatively small fraction of the initial population, with most particles being ejected from the solar system by planetary perturbations. Using $d N(q)$ with
$0 \leqslant \gamma \leqslant 1$ (Section 2.1), $a \sim 10^{3}$ AU and isotropic distribution of inclination vectors, roughly $0.8 \%-1.5 \%$ of particles with $D=100 \mu \mathrm{m}$ evolve down into the inner solar system and decouple from Jupiter (as defined by the aphelion distance $Q=a(1+e)<4 \mathrm{AU})$, without being disrupted by an impact (with standard $S_{1}=S_{2}=0$; Section 2.3) or ever having $q<0.05 \mathrm{AU}$ (to avoid sublimation). These particles can potentially be important for the terrestrial impact record and interpretation of the meteor radar data. The bulk of OCC particles that do not reach $a \sim 1 \mathrm{AU}$, do not significantly contribute to Earth's accretion, because these particles spend most of their lifetimes at $R \gg 1 \mathrm{AU}$.

The fraction of OCC particles reaching $a \sim 1 \mathrm{AU}, f_{1}$, normalized to the number of particles whose orbits were initially bound (see Section 2.1), is sensitive to particle size. For $D=10 \mu \mathrm{m}, d N(q)$ described in Section 2.1, and initial $a \sim 10^{3} \mathrm{AU}, f_{1} \simeq 0.15-0.2$. For $D=300 \mu \mathrm{m}$, on the other hand, $f_{1} \simeq 2 \times 10^{-3}$. Moreover, for $D=1 \mathrm{~mm}$, only one particle out of the total of 5000 reached $a \sim 1$ AU. This trend, with the larger particles having progressively smaller $f_{1}$ values, has interesting implications for the observations of sporadic meteors (Section 4).

The above estimates used $\tau_{\text {coll }}$ as described in Section 2.3.2. Collisional disruption, however, turned out to have only a modest effect for the standard G85 $\tau_{\text {coll }}\left(S_{1}=S_{2}=0\right)$ and the particle sizes considered here. For example, only $\sim 2 \%$ of particles with $D=100 \mu \mathrm{m}$ that reached $a \sim 1 \mathrm{AU}$ in our numerical integration have disrupted prior to decoupling from Jupiter, as detected in post-processing of the integration output, with the standard G85 $\tau_{\text {coll }}$. A great majority of particles with $D=300 \mu \mathrm{m}$ also survived. The effect of disruptive collisions becomes more significant for $D \sim 1 \mathrm{~mm}$, for which $\tau_{\text {coll }}$ is significantly shorter (Section 2.3.2) and P-R drag is weaker.

The thermal effects discussed in Section 2.3.1 turned out to be very important for all particle sizes considered here. For example, 75 out of 122 particles (i.e., over 60\%) with 


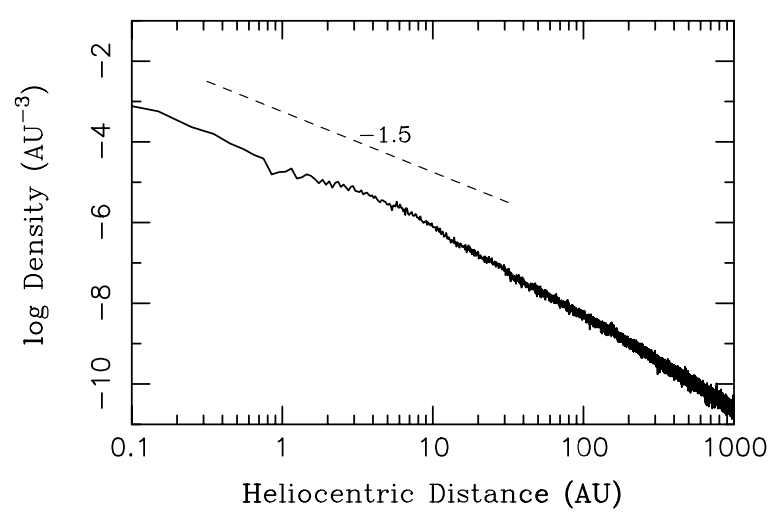

Figure 4. Radial distribution of OCC particles that we obtained in our model. The number density was normalized to the input flux of one particle released on bound orbit per year. We used $D=100 \mu \mathrm{m}$ here. The distribution with $D=300 \mu \mathrm{m}$ looks similar for $R>5 \mathrm{AU}$, but is depleted below $5 \mathrm{AU}$, relative to the one shown here, because fewer particles with $D=300 \mu \mathrm{m}$ are able to decouple from Jupiter. Particles were released on orbits with $a \sim 10^{3} \mathrm{AU}$ and uniformly random $\sin i$. The initial distribution $d N(q)$ was set as described in Section 2.1 with $\gamma=0.5$ (the results are not sensitive to $\gamma$ ).

$D=100 \mu \mathrm{m}$ that ever reached $Q<4 \mathrm{AU}$, previously had $q<0.05 \mathrm{AU}$, which is our crude threshold for the thermal destruction of particles. Also, 68 out of $74 D=300 \mu \mathrm{m}$ particles (over 90\%) reaching $Q<4$ AU previously had $q<0.05$ AU. The thermally destroyed particles are removed and the orbital histories after their disruption are not used for our analysis.

These fractions are a direct consequence of the relative importance of planetary perturbations and P-R drag on particles with different $D$ and $q$. The orbital evolution of a large particle on the OCC-like orbit is primarily controlled by planetary perturbations. Sooner or later, the planets will eject the particle from the solar system, unless the orbit shrinks and decouples from Jupiter. To achieve this, $q$ of the particle's orbit must be very low, so that the P-R drag timescale is short (see Figure 2(b)). But if $q$ is low, it may easily drop below $q<0.05 \mathrm{AU}$, where the particle is removed, thus explaining why most large particles reaching $Q<4$ AU previously have $q<0.05$ AU.

Interestingly, the fraction of particles with $D \simeq 100-300 \mu \mathrm{m}$ reaching $a \sim 1$ AU is not overly sensitive to the initial perihelion distance, as far as $q \lesssim 5 \mathrm{AU}$. This is because it is more likely to decouple if $q$ is low, because P-R drag is stronger, but this trend is nearly canceled, because particles with very low $q$ tend to drop below $q=0.05 \mathrm{AU}$ and sublimate before they can decouple. Particles with $D \simeq 100-300 \mu \mathrm{m}$ and $q>5 \mathrm{AU}$, on the other hand, tend to have very long P-R drag timescales (e.g., $\tau_{\text {fall }}>50$ Myr for initial $a=10^{3}$ AU; Figure 2(b)), and are scattered by planets from the solar system.

\subsection{Orbital and Spatial Distributions}

Here we discuss the expected distribution of OCC particles in the inner solar system. Figure 4 shows the number density of OCC particles as a function of $R$. As expected, the particle density increases toward the Sun. The radial distribution of $D=100 \mu \mathrm{m}$ particles can be approximated by a power law, $d N(R) \propto R^{-\alpha} d R$, where $\alpha \simeq 1.5$ for $R<5 \mathrm{AU}$, and $\alpha \simeq 2.0$ for $R>10$ AU. Both these radial dependencies are significantly steeper than $d N(R) \propto R^{-1} d R$, expected for distribution of particles on nearly circular orbits (see, e.g., Dermott et al. 2001).

The relatively steep radial distribution is a consequence of Keplerian motion of particles with $e \sim 1$. For $R<a$, the time
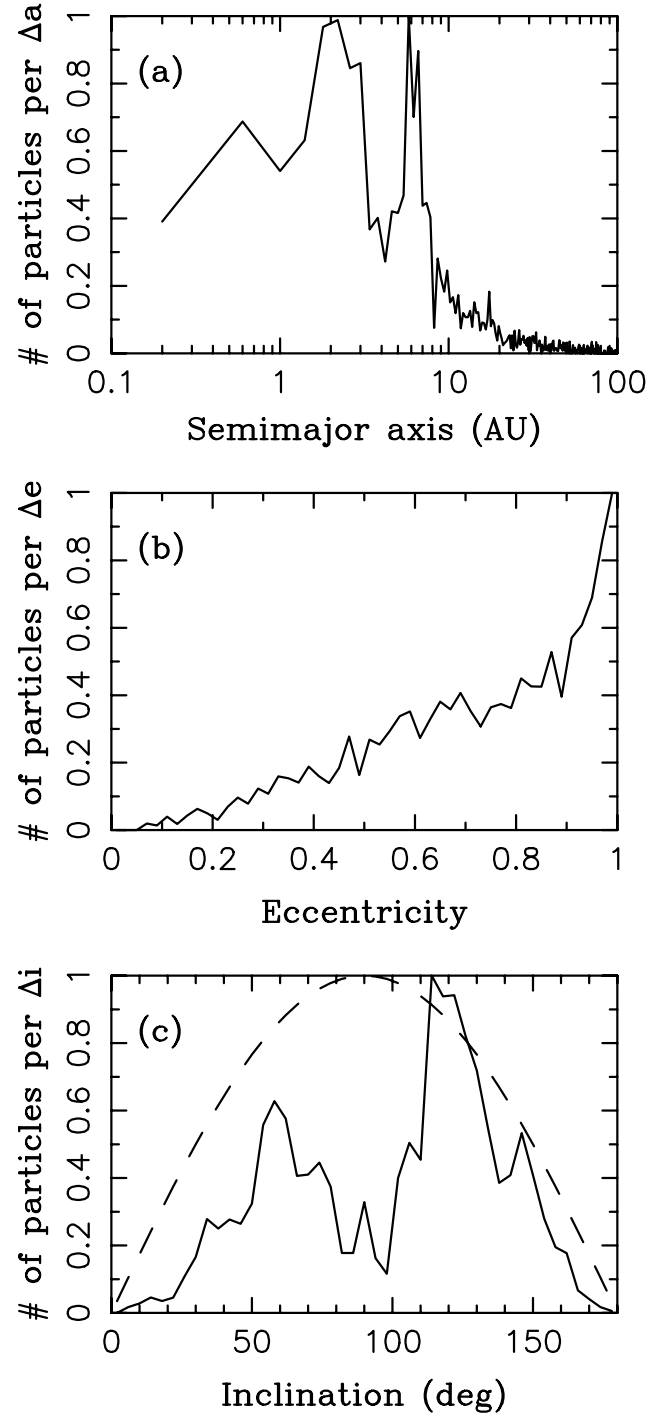

Figure 5. Steady state distribution of orbital elements of particles with $D=100 \mu \mathrm{m}$ : (a) $d N(a)$, (b) $d N(e)$, and (c) $d N(i)$. The distributions shown here ignore particles with $R>5 \mathrm{AU}$. They therefore represent the steady state distribution of orbits in the inner solar system, which is relevant for observations of the inner zodiacal cloud and sporadic meteors.

spent by a particle on the Keplerian orbit between $R$ and $R+d R$ is $d t \propto R^{0.5} d R$. This leads to $d N(R) \propto R^{-1.5} d R$, if the expected number of particles, which is proportional to $d t$, is divided by volume $4 \pi R^{2} d R$. For $R>a$, on the other hand, appropriate for large radial distances, $d t \sim$ const. and $d N(R) \propto R^{-2} d R$. Thus, $\alpha \simeq 1.5-2$ is expected for $e \sim 1$ (see also Liou et al. 1999).

We find that the number density of OCC particles at $R \sim$ $1 \mathrm{AU}$ is mainly contributed by the particles that orbitally decoupled from Jupiter. This shows the importance of orbital decoupling for the distribution of OCC particles in the inner solar system, and their accretion by the Earth. Specifically, most OCC particles accreted by the Earth are expected to have $a(1+e)<4 \mathrm{AU}$ despite the fact that their orbits started with $a \gtrsim 10^{3}$ AU.

Figure 5 shows the distributions of orbital elements for OCC particles with $D=100 \mu \mathrm{m}$ and $R<5$ AU. The semimajor axis distribution, $d N(a)$, has a broad maximum centered at $0.5-2 \mathrm{AU}$ with $a \sim 2$ AU being the most common. $d N(a)$ decreases toward larger $a$, because particles with $a>3$ AU are coupled to Jupiter, have short dynamical lifetimes, and do not spend 
much time at $R<5 \mathrm{AU}$. The peak of $d N(a)$ at $a=6-7 \mathrm{AU}$ is produced by orbits in the exterior mean motion resonances with Jupiter, which prolong the dynamical lifetime of particles by phase protecting them against encounters with Jupiter (Liou et al. 1999).

The eccentricity distribution $d N(e)$ increases toward $e \sim 1$, which is expected because all orbits started with $e>0.995$. The tail extending to the moderate- and low-eccentricity values is due to the dynamically long-lived particles, whose orbits decouple from Jupiter and become circularized by P-R drag.

The inclination distribution is also interesting as it significantly deviates from the initial distribution with $d N(i) \propto$ $\sin (i) d i$ (Figure 5(c)). The retrograde orbits are more common than the prograde ones. The preference for retrograde orbits is probably caused by gravitational perturbations from Jupiter that are more effective on prograde orbits, because the encounter speeds are lower, and thus $\Delta V \mathrm{~s}$ are larger. The prograde particles should therefore have shorter dynamical lifetimes than the retrograde particles, which would explain their relative paucity in a steady state $d N(i)$ for $R<5$ AU. Interestingly, however, the particles that decouple from Jupiter and reach $a \sim 1$ AU by $\mathrm{P}-\mathrm{R}$ drag do not show a strong preference for retrograde orbits.

In addition, the steady state $d N(i)$ lacks orbits with $i \sim 90^{\circ}$. We believe that this is a consequence of Kozai dynamics (e.g., Kozai 1962). It is well known that the initially near-polar orbits will suffer large oscillations of $q$ driven by variations of the orbital angular momentum vector. Most of these orbits can therefore reach very low $q$ values, where the particles will be destroyed by thermal effects. Indeed, by studying the orbital histories of particles that started with $i \sim 90^{\circ}$, we found that most of these orbits had $q<0.05 \mathrm{AU}$ prior to reaching $Q<4$ AU.

The steady state distribution of orbital elements of $D=$ $300 \mu \mathrm{m}$ particles is similar to the one discussed above. Instead of having a single peak at $a=6-7$ AU, however, $d N(a)$ for $a>5 \mathrm{AU}$ is more irregular showing many peaks and dips. Apparently, since the larger $D=300 \mu \mathrm{m}$ particles drift more slowly by P-R drag, they are more susceptible to capture in a large number of resonances (Liou et al. 1999). The second difference concerns $d N(e)$, which is slightly more clumped toward $e \sim 1$ for $D=300 \mu \mathrm{m}$ than for $D=100 \mu \mathrm{m}$.

\subsection{Radiants and Orbits of Particles Accreted by the Earth}

The radiants of OCC particles are located near Earth's apex (Figure 6). This is logical because the retrograde OCC particles, which come from the apex direction, have much higher velocity relative to the Earth $\left(\sim 60 \mathrm{~km} \mathrm{~s}^{-1}\right)$ than particles on prograde orbits. The retrograde particles therefore also have, according to the usual $n \sigma v$ rule, rather large impact probabilities with the Earth. In addition, the ionization cutoff used here (Equation (9)) poses a rather strict limit on the mass of meteoroids that can be detected by SMRs at low speeds. For example, a $D=100 \mu \mathrm{m}$ particle with $m=10^{-6} \mathrm{~g}$ and $v_{g}=30 \mathrm{~km} \mathrm{~s}^{-1}$ has $I=0.01$, which is near or slightly above the detection limit of AMOR, and way below the detection limit of CMOR.

The model radiants form the south and north apex concentrations, just as observed (e.g., Jones \& Brown 1993; Galligan \& Baggaley 2005; Campbell-Brown 2008). The lack of radiants within $\sim 10^{\circ}$ about the ecliptic is due to near absence of OCC meteoroids with $i \simeq 180^{\circ}$ (see Figure 5(c)). The lack of radiants with $b>50^{\circ}$ (or $b<-50^{\circ}$ ) is the consequence of the inclination distribution shown in Figure 5(c) that is depleted in orbits with $i \sim 90^{\circ}$.
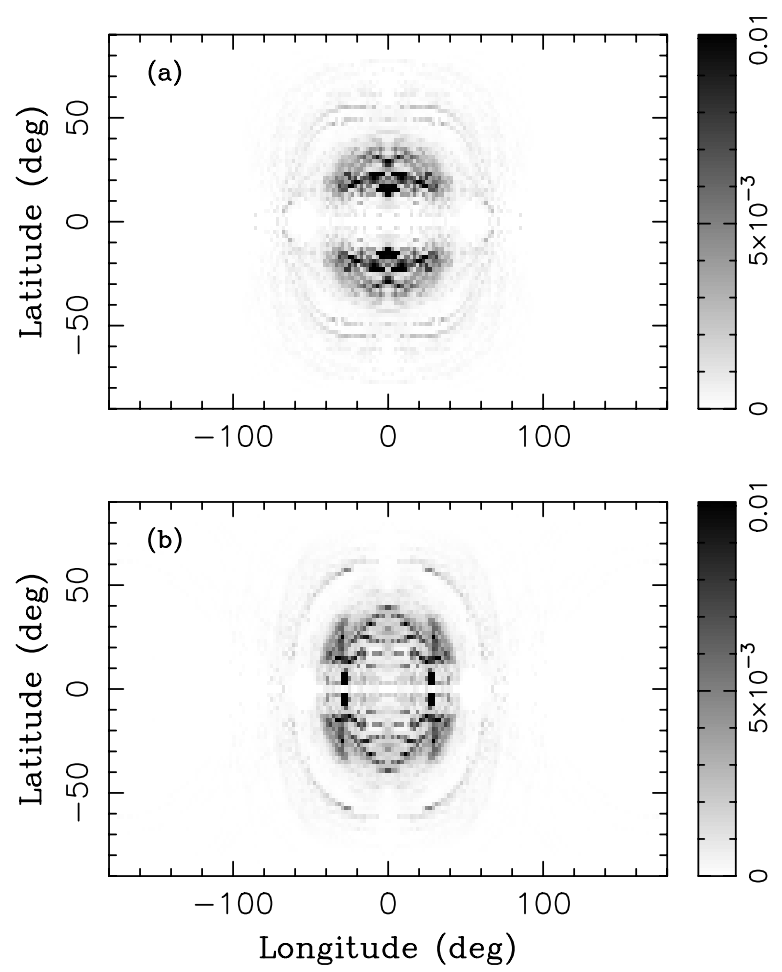

Figure 6. Radiants of OCC meteoroids determined in our model with $I^{*}=0.01$ : (a) $D=100 \mu \mathrm{m}$ and (b) $D=300 \mu \mathrm{m}$. The north and south apex sources are clearly visible. In addition, the radiant distribution in panel (b) shows a ring structure centered at $(l, b)=(0,0)$ and extending to $\simeq 60^{\circ}$ in $l$ and $b$. The high-frequency fluctuation of radiant density between neighbor bins is due to insufficient statistics and should be disregarded. The units of the side bar are arbitrary.

Most north (south) radiants fall into an area on the north (south) hemisphere that has the characteristic triangular or half-disk shape. For $D=100 \mu \mathrm{m}$, the centers of radiant concentrations are at $b \simeq \pm 20^{\circ}$. For $D=300 \mu \mathrm{m}$, the centers are at $b \simeq \pm 25^{\circ}$. This reflects the differences in $d N(i)$ between the populations of particles with $D=100 \mu \mathrm{m}$ and $D=300 \mu \mathrm{m}$ that we obtained in the model. For comparison, observations indicate that $b \simeq \pm 15^{\circ}$ (e.g., Galligan \& Baggaley 2005; Chau et al. 2007; Campbell-Brown 2008). In addition, the apex sources that we obtained in our model tend to be more stretched in both $l$ and $b$ than the observed ones.

While more modeling work will be needed to test things with a better statistic, the issues discussed above may indicate that a better fit to observations could be obtained if the retrograde source had inclinations closer to $180^{\circ}$ than the bulk of OCC particles with $100^{\circ}<i<160^{\circ}$. It is not clear how this could be achieved by tweaking the parameters of our model. Instead, clues such as these seem to highlight the importance of known HTCs. Indeed, the two prominent active HTCs, 1P/Halley and 55P/Tempel-Tuttle, both have $i \simeq 162^{\circ}$. They would therefore be expected to produce apex sources closer to the ecliptic than the bulk of retrograde OCC particles (Figure 7).

An interesting feature in Figure 6(b) is the presence of a ring that stretches to $\pm 60^{\circ}$ in longitude and latitude. A similar ring has been noted in Campbell-Brown (2008), who suggested that the region inside the ring can be depleted in meteor radiants, except for apex sources, because the retrograde meteoroids with radiants inside the ring would have shorter collisional lifetimes. Our simple collisional model cannot reproduce this effect because $\tau_{\text {coll }}$ is independent of $i$ (see Section 2.3.2). In 


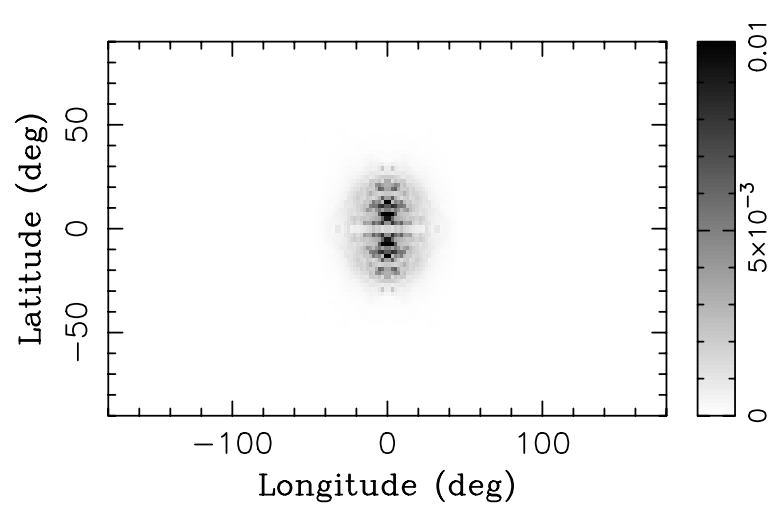

Figure 7. Same as Figure 6 but for $D=100 \mu \mathrm{m}$ particles released from $1 \mathrm{P} /$ Halley. The results for $55 \mathrm{P} /$ Tempel-Tuttle are similar.

addition, W09 suggested that the origin of the ring can be traced back to Kozai dynamics, which confines the allowed radiants of particles on high-inclination orbits. Here we confirm the W09 result by isolating particles that contribute to the ring, and checking on their orbital behavior.

The impact speed of OCC particles peaks at $v_{g} \simeq 60 \mathrm{~km} \mathrm{~s}^{-1}$ (Figure 8 for $D=100 \mu \mathrm{m}$ and Figure 9 for $D=300 \mu \mathrm{m}$ ), which is a nice match to observations of apex meteors (Galligan \& Baggaley 2005; Campbell-Brown 2008). ${ }^{7}$ The width of the peak also looks good (see Figure 13 in Campbell-Brown 2008). In comparison, using a population of meteoroids from $1 \mathrm{P} /$ Halley and 55P/Tempel-Tuttle, W09 obtained a peak at $v_{g} \simeq 70 \mathrm{~km} \mathrm{~s}^{-1}$, which is expected for large particles that have not evolved far from their parent comet orbit.

Figures 8 and 9 also show the model distributions of orbital elements of OCC meteoroids. Distributions $d N(a)$, now heavily weighted by the collision probability (see Figure 5), peak at $a \simeq 1 \mathrm{AU}$ and show a tail extending to $a>2 \mathrm{AU}$. The

\footnotetext{
7 Note that observations by HPLA radars, such as AO or ALTAIR, measure the apex peak speed at $\simeq 55 \mathrm{~km} \mathrm{~s}^{-1}$.
}
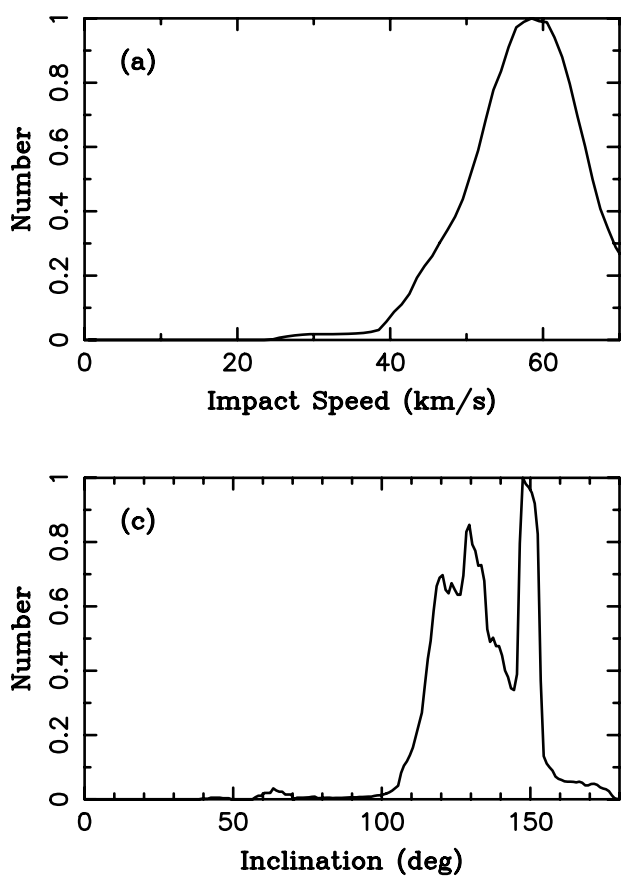

meteoroids in the peak have orbits that have strongly evolved by P-R drag. The model distributions for $D=100 \mu \mathrm{m}$ and $D=300 \mu \mathrm{m}$ are similar, except for a few wiggles produced by statistical fluctuations. Both provide a good match to the observed semimajor axis distribution of apex meteors (see Figure 11 in Galligan \& Baggaley 2005).

According to our model, most apex meteors should have inclinations between $i \sim 100^{\circ}$ and $180^{\circ}$ (Figures 8(c) and 9(c)), which is also the range indicated by observations. Unlike $d N(i)$ measured by radars, which shows a broad peak centered at $i \sim 150^{\circ}-160^{\circ}$, our model $d N(i)$ is more spread and noisy. While part of this discrepancy could be blamed on insufficient statistics in our model, it may also point to a more fundamental problem.

The eccentricity distribution is puzzling. For both $D=$ $100 \mu \mathrm{m}$ and $D=300 \mu \mathrm{m}$, we obtained $d N(e)$ that raises toward $e \sim 1$. This trend is slightly more pronounced for $D=300 \mu \mathrm{m}$ (Figure 9(d)) than for $D=100 \mu \mathrm{m}$ (Figure 8(d)). W09, using selected HTCs for parent bodies of apex meteors, obtained $d N(e)$ that also peaked toward $e \sim 1$. In contrast, the observed apex meteors have nearly flat $d N(e)$ at $0.2<e<1$, and show a slight depletion for $e<0.1$ (Figure 13 in Campbell-Brown 2008).

The cause of these differences is unclear. To obtain lower values of $e$ in our model, the orbits would need to become more circularized by P-R drag before arriving to $a \sim 1 \mathrm{AU}$. This could be achieved, for example, if more weight is given to particles starting with $q>1 \mathrm{AU}$. We confirm this by using $\gamma>1$, but a detailed match to the observed eccentricity distribution remains elusive. A detailed analysis of this problem is left for future studies.

\section{RELATIVE IMPORTANCE OF HELION/ANTIHELION AND APEX SOURCES}

The observations of sporadic meteors show that the relative importance of helion/antihelion and apex sources depends on
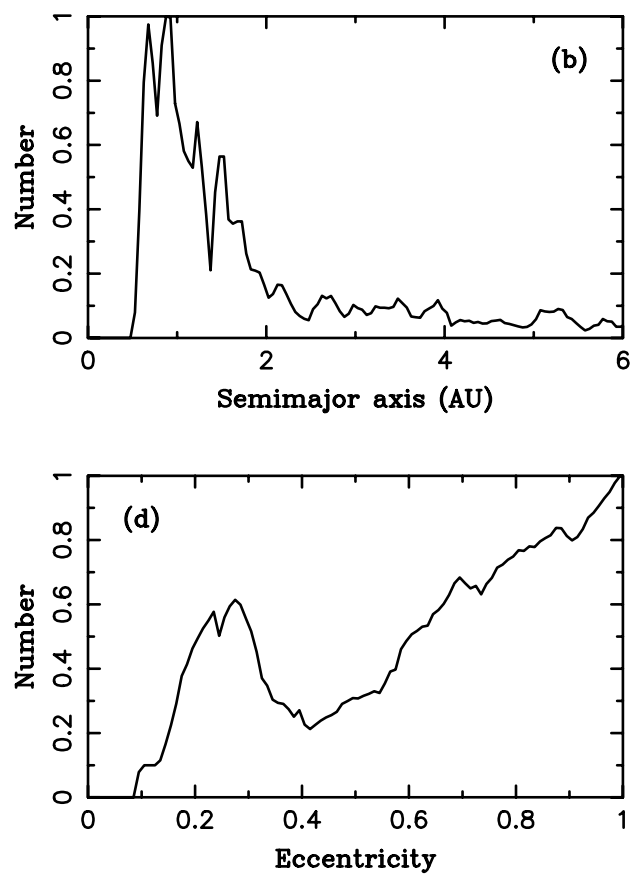

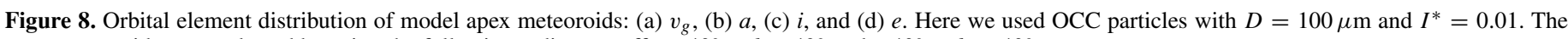
apex meteoroids were selected by using the following radiant cutoffs: $-40^{\circ}<l<40^{\circ}$ and $-40^{\circ}<b<40^{\circ}$. 

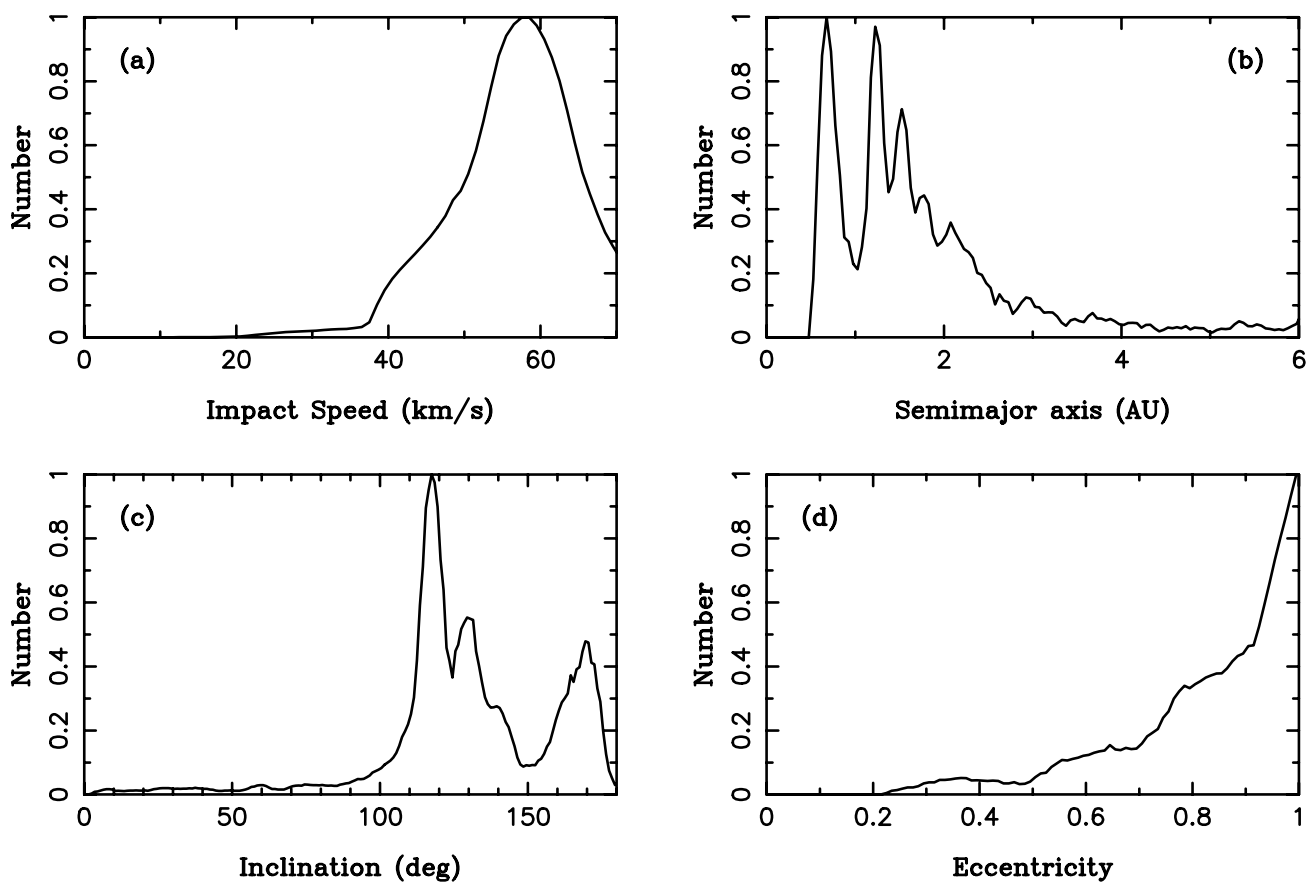

Figure 9. Same as Figure 8 but for $D=300 \mu \mathrm{m}$ OCC particles.

the sensitivity of the radar that is used to carry out such observations. The less sensitive SMRs with $I^{*} \sim 1$, such as the Harvard Radar Meteor Project (HRMP; Jones \& Brown 1993; Taylor \& Elford 1998) or CMOR, detect 3-10 times more helion/antihelion meteors then apex meteors (see, e.g., Campbell-Brown 2008 for comparison of different radars). The more sensitive radars, such as AMOR with $I^{*} \sim 0.001-0.01$, on the other hand, detect a relatively larger number of apex meteors. Finally, the apex meteors are predominant in observations by the highly sensitive AO radar (e.g., Janches et al. 2003) because of their ability to detect small particles (Fentzke \& Janches 2008; Fentzke et al. 2009).

This trend can be explained if the size frequency distribution (SFD) of apex meteoroids is steeper (i.e., if the number of meteoroids increases more sharply with decreasing size) than that of the helion/antihelion meteoroids because radars that are capable of detecting smaller meteoroids would then be expected to see many more apex meteors (Fentzke \& Janches 2008). For example, the initial SFD of particles produced by disrupted OCCs (or HTCs) could be steeper than the one produced by the sources of the helion/antihelion meteoroids (presumably active and disrupted JFCs; W09; Nesvorný et al. 2010). While this is a possibility that cannot be ruled out by the existing data, there are also no indications that this might be true (McDonnell et al. 1987; Grün et al. 2001; Green et al. 2004; Reach et al. 2007).

We propose that the predominance of apex meteors in $\mathrm{AO}$ observations is caused by orbital dynamics of particles released from OCCs (or HTCs). Let us assume that the initial SFD of particles released from OCCs (or HTCs) is $d N_{0}(D)=$ $N_{0} D^{-\zeta} d D$. The SFD of meteoroids accreted by the Earth will then be

$$
d N(D)=f_{0} P_{\mathrm{i}} N_{0} D^{-\zeta} d D
$$

where $f_{0}$ is the fraction of particles that remain on bound orbits (see Section 2.1) and $P_{\mathrm{i}}$ is the impact probability of these particles on the Earth. Factor $f_{0}$ expresses the removal of small particles by radiation pressure (see Section 2.1 ). Thus, the size dependence of $f_{0}$ is such that it cannot increase the number of small particles relative to the large ones. ${ }^{8}$

Table 1 lists $P_{\mathrm{i}}$ and $v_{\infty}$ for various sources. For example, the OCC particles with $D=30 \mu \mathrm{m}$ and $D=300 \mu \mathrm{m}$ have $P_{\mathrm{i}}=$ $2 \times 10^{-6}$ and $P_{\mathrm{i}}=5 \times 10^{-7}$, respectively. This indicates that $d N(D)$ should have a steeper slope than $d N_{0}(D)$. Specifically, if $d N_{0}(D)$ can be approximated by $D^{-\zeta} d D$ for $D \sim 30-300 \mu \mathrm{m}$, where $\zeta$ is a constant, we find that $d N(D) \propto D^{-(\zeta+\delta)} d D$, where $\delta \sim 0.6$ for OCCs. This estimate was obtained with $0 \leqslant \gamma \leqslant 1$, $S_{1} \sim S_{2} \sim 0, q^{*}=0.05 \mathrm{AU}$, and initial $a \sim 10^{3} \mathrm{AU}$. The results for $S_{2}=1$, applicable if $\tau_{\text {coll }}$ were $\sim 10$ times longer than in G85, and for HTCs are similar.

For comparison, W09 suggested that comet 2P/Encke (or an orbitally similar lost comet) is the main source of helion/antihelion meteors. We find that particles released from comet $2 \mathrm{P} /$ Encke have $P_{\mathrm{i}}=6 \times 10^{-5}$ for $D=30 \mu \mathrm{m}$ and $P_{\mathrm{i}}=2.3 \times 10^{-4}$ for $D=300 \mu \mathrm{m}$ (Table 1). If these estimates are representative for the sources of the helion/antihelion meteors, they suggest that the slope of $d N(D)$ should be shallower than that of $d N_{0}(D)(\delta \sim-0.6)$.

We therefore find that $d N(D)$ of apex meteoroids is expected to be steeper than that of helion/antihelion meteoroids, even if the initial SFD of particles released from the respective sources-JFCs and OCCs/HTCs-were similar. This effect is produced by orbital dynamics of particles starting on different initial orbits. As we discussed in Section 3.1, the Earth-impact record of OCC particles is mainly contributed by those particle that decouple from Jupiter. Since the decoupling efficiency, described by factor $f_{1}$ in Section 3.1, ramps up toward smaller $D$, the population of small OCC (or HTC) particles is enhanced, relative to large ones. This effect is weaker for JFC meteoroids, for which the correlation between $f_{1}$ and $P_{\mathrm{i}}$ is not as extreme. In the JFC case, the population of small particles accreted by

\footnotetext{
8 Factor $f_{0}(D)$ could presumably be approximated by a step function with $f_{0}(D)=0$ for $D<D^{*}$ and $f_{0}(D)=1$ for $D>D^{*}$, where $D^{*}$ is the critical diameter implied by Equation (2).
} 
Table 1

The Earth Impact Probability, $P_{\mathrm{i}}$, and Mean Impact Speed, $\left\langle v_{\infty}\right\rangle$, of Particles Released on Different Orbits

\begin{tabular}{|c|c|c|}
\hline $\begin{array}{l}D \\
(\mu \mathrm{m})\end{array}$ & $\begin{array}{c}P_{\mathrm{i}} \\
\left(10^{-6}\right)\end{array}$ & $\begin{array}{c}\left\langle v_{\infty}\right\rangle \\
\left(\mathrm{km} \mathrm{s}^{-1}\right)\end{array}$ \\
\hline \multicolumn{3}{|c|}{ OCCs } \\
\hline 10 & $4.6(4.6)$ & $52(52)$ \\
\hline 30 & $1.9(1.9)$ & $47(47)$ \\
\hline 100 & $1.0(1.4)$ & $46(46)$ \\
\hline 300 & $0.5(0.6)$ & $55(54)$ \\
\hline 1000 & $0.2(0.3)$ & $58(58)$ \\
\hline \multicolumn{3}{|c|}{ 1P/Halley (HTC) } \\
\hline 10 & $57(57)$ & $60(60)$ \\
\hline 30 & $100(110)$ & $59(59)$ \\
\hline 100 & $80(110)$ & $59(59)$ \\
\hline 300 & $24(46)$ & $65(61)$ \\
\hline 1000 & $17(22)$ & $67(67)$ \\
\hline \multicolumn{3}{|c|}{ 55P/Tempel-Tuttle (HTC) } \\
\hline 10 & $53(53)$ & $60(60)$ \\
\hline 30 & $120(120)$ & $59(59)$ \\
\hline 100 & $80(120)$ & $60(59)$ \\
\hline 300 & $27(51)$ & $66(61)$ \\
\hline 1000 & $16(23)$ & $68(66)$ \\
\hline \multicolumn{3}{|c|}{ 2P/Encke (JFC) } \\
\hline 10 & $17(17)$ & $18.0(18.0$ \\
\hline 30 & $60(60)$ & $18.5(18.5$ \\
\hline 100 & $210(220)$ & $18.7(18.4$ \\
\hline 300 & $230(340)$ & $23.6(22.0$ \\
\hline 1000 & $120(300)$ & $30.5(27.0$ \\
\hline
\end{tabular}

Notes. For each particle's diameter, $D$, we give our best estimate values for the standard G85 $\tau_{\text {coll }}\left(S_{1}=S_{2}=0\right.$ ), and for $S_{1}=0$ and $S_{2}=1$ (values in parenthesis). The longer collisional lifetime in the later case leads to the larger $P_{\mathrm{i}}$ values. The effect of disruptive collisions is significant for $D \gtrsim 300 \mu \mathrm{m}$.

the Earth is suppressed by their short P-R drag timescale, and consequently, lower $P_{\mathrm{i}}$.

The magnitude of the SFD effects discussed above is just right to explain observations. If the ionization threshold $I^{*}$ of AMOR is $\sim 100$ times lower than that of CMOR/HRMP, these more sensitive instruments are expected to detect meteoroids that are $\sim 5$ times smaller in size. If they detect 3-10 times more apex meteors than the helion/antihelion meteors (e.g., Campbell-Brown 2008), this would suggest that the SFD slope index difference between apex and helion/antihelion meteors is $\delta \sim 0.7-1.4$. We found $\delta \sim 1.2$ above, in good agreement with observations. Figure 10 illustrates the relative strength of sporadic meteor sources expected from our model.

\section{CONCLUSIONS}

We found that only a very small fraction, $f_{1} \lesssim 10^{-4}$, of $D \gtrsim 1 \mathrm{~mm}$ OCC particles can ever make it into the inner solar system. The relevance of these very large OCC particles to observations of sporadic meteors is therefore not obvious. The situation looks more favorable for OCC particles with $D \simeq 100-300 \mu \mathrm{m}$. These particles should survive the effects of radiation pressure, if released from returning OCCs at $R \gtrsim 4-12$ AU. Moreover, about $0.2 \%-1.5 \%$ avoid being collisionally disrupted or thermally destroyed, decouple from Jupiter, and finally spiral down to $a \sim 1$ AU, where their Earthimpact probability is increased by orders of magnitude.

We estimated that the overall probability of Earth impact per one particle released on bound orbit from the returning OCC

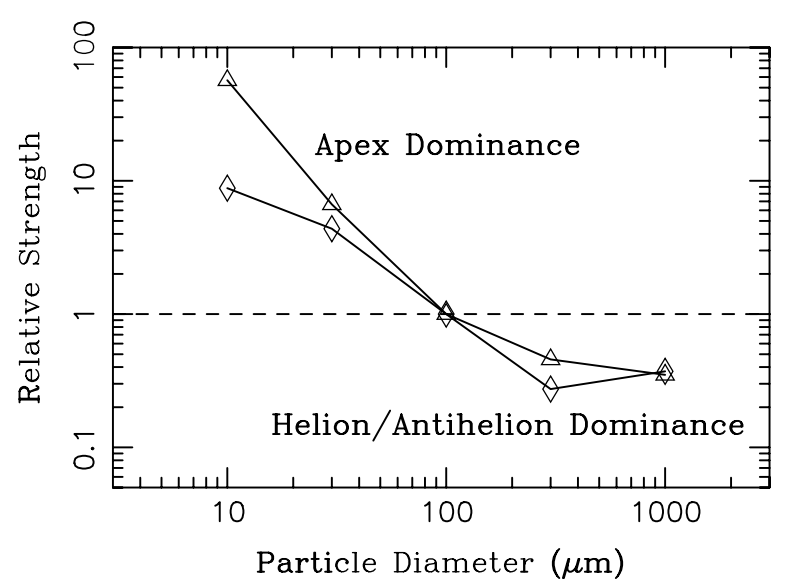

Figure 10. Expected relative strength of the apex and helion/antihelion sources as a function of particle size. The triangles (diamonds) show the result for OCC (1P/Halley) particles. We calculated the relative strength by dividing $P_{\mathrm{i}}(D)$ listed in Table 1 for OCC (1P/Halley) particles (assumed here to represent the apex source) by $P_{\mathrm{i}}(D)$ of $2 \mathrm{P} /$ Encke particles (assumed to represent the helion/ antihelion source; W09). The $P_{\mathrm{i}}(D)$ ratio is a proxy for the relative strength of meteor sources if $d N_{0}(D)$ of different initial populations had roughly the same shape. We arbitrarily normalized the ratio to 1 for particles with $D=100 \mu \mathrm{m}$. As more sensitive radars detect smaller particles, they are expected see more apex meteors, because $P_{\mathrm{i}}(D)$ of OCC (1P/Halley) particles increases while that of JFC particles drops.

is $P_{\mathrm{i}} \sim 0.5-1 \times 10^{-6}$ for $D \simeq 100-300 \mu \mathrm{m}$. This is $50-80$ times lower than $P_{\mathrm{i}}$ expected for particles released from HTCs such as 1P/Halley and 55P/Tempel-Tuttle, and 200-400 times lower than $P_{\mathrm{i}}$ expected for JFCs such as $1 \mathrm{P} /$ Encke. The OCC particles will therefore significantly contribute to the sporadic meteor complex only if the mass of material produced by disrupting OCCs is large enough to compensate for these factors. From L02, we can roughly estimate that $\sim 5$ returning OCCs disrupt per year producing the mass input of perhaps as much as $\sim 10^{18} \mathrm{~g} \mathrm{yr}^{-1}$, or $3 \times 10^{8} \mathrm{~kg} \mathrm{~s}^{-1}$. Only a small fraction of this mass will end in bound particles with $D \simeq 100-300 \mu \mathrm{m}$. For comparison, the active JFCs produce $\sim 300 \mathrm{~kg} \mathrm{~s}^{-1}$ (Reach et al. 2007).

We found that the SFD of apex meteoroids, presumably starting on highly eccentric orbits, is expected to be steeper than those of helion/antihelion meteoroids, even if their initial SFDs were similar. The steepening of the SFD slope of apex meteoroids results from the efficiency with which OCC/HTC meteoroids of different sizes decouple from Jupiter. This result has interesting implications for observations of sporadic meteors because it can explain why the north/south apex sources are more represented in observations of highly sensitive radars that are capable of detecting smaller meteoroids.

This article is based on work supported by the NASA's PG\&G program. The work of D.V. was partially supported by the Czech Grant Agency (grant 205/08/0064) and the Research Program MSM0021620860 of the Czech Ministry of Education. The work of D.J. was partially supported by NSF Award AST 0908118. We thank W. F. Bottke for sharing with us his Öpik code, M. Campbell-Brown for useful discussions, and Tadeusz Jopek for a very helpful review of this article.

\section{REFERENCES}

Bottke, W. F., Nolan, M. C., Greenberg, R., \& Kolvoord, R. A. 1994, Icarus, 107,255

Burns, J. A., Lamy, P. L., \& Soter, S. 1979, Icarus, 40, 1 
Breiter, S., \& Jackson, A. A. 1998, MNRAS, 299, 237

Campbell-Brown, M. D. 2008, Icarus, 196, 144

Čapek, D., \& Borovička, J. 2009, Icarus, 202, 361

Chau, J. L., Woodman, R. F., \& Galindo, F. 2007, Icarus, 188, 162

Choi, Y.-J., Weissman, P., Chesley, S., et al. 2006, CBET, 563, 1

Dermott, S. F., Grogan, K., Durda, D. D., et al. 2001, in Interplanetary Dust, ed. E. Grün, B. S. Gustafson, S. Dermott, \& H. Fechtig (Berlin: Springer), 569

Dikarev, V., Grün, E., Baggaley, J., et al. 2005, Adv. Space Res., 35, 1282

Drolshagen, G., Dikarev, V., Landgraf, M., Krag, H., \& Kuiper, W. 2008, Earth Moon Planets, 102, 191

Duschl, W. J., Gail, H.-P., \& Tscharnuter, W. M. 1996, A\&A, 312, 624

Dybczyński, P. A. 2001, A\&A, 375, 643

Fentzke, J. T., \& Janches, D. 2008, J. Geophys. Res. (Space Phys.), 113, 3304

Fentzke, J. T., Janches, D., \& Sparks, J. J. 2009, J. Atmos. Sol.-Terr. Phys., 71, 653

Francis, P. J. 2005, ApJ, 635, 1348

Galligan, D. P., \& Baggaley, W. J. 2004, MNRAS, 353, 422

Galligan, D. P., \& Baggaley, W. J. 2005, MNRAS, 359, 551

Green, S. F., McDonnell, J. A. M., McBride, N., et al. 2004, J. Geophys. Res., 109,12

Greenberg, R. 1982, AJ, 87, 184

Grün, E., Hanner, M. S., Peschke, S. B., et al. 2001, A\&A, 377, 1098

Grün, E., Zook, H. A., Fechtig, H., \& Giese, R. H. 1985, Icarus, 62, 244

Henning, T., \& Mutschke, H. 1997, A\&A, 327, 743

Janches, D., \& Chau, J. L. 2005, J. Atmos. Sol.-Terr. Phys., 67, 1196

Janches, D., Close, S., \& Fentzke, J. T. 2008, Icarus, 193, 105

Janches, D., Heinselman, C. J., Chau, J. L., Chandran, A., \& Woodman, R. 2006, J. Geophys. Res. (Space Phys.), 111, 7317

Janches, D., Nolan, M. C., Meisel, D. D., et al. 2003, J. Geophys. Res. (Space Phys.), 108, 1222

Jenniskens, P. 2008, Earth Moon Planets, 102, 505

Jones, J., \& Brown, P. 1993, MNRAS, 265, 524
Jones, J., Campbell, M., \& Nikolova, S. 2001, in Meteoroids 2001 Conference, ed. B. Warmbein (ESA Special Publication, Vol. 495; Noordwijk: ESA), 575

Kasuga, T., Yamamoto, T., Kimura, H., \& Watanabe, J. 2006, A\&A, 453, L17

Kessler-Silacci, J. E., Dullemond, C. P., Augereau, J.-C., et al. 2007, ApJ, 659, 680

Kozai, Y. 1962, AJ, 67, 591

Kresak, L. 1976, Bull. Astron. Inst. Czech., 27, 35

Levison, H. F., \& Duncan, M. J. 1994, Icarus, 108, 18

Levison, H. F., Morbidelli, A., Dones, L., et al. 2002, Science, 296, 2212

Liou, J.-C., Zook, H. A., \& Jackson, A. A. 1999, Icarus, 141, 13

McDonnell, J. A. M., Evans, G. C., Evans, S. T., et al. 1987, A\&A, 187, 719

Moro-Martín, A., \& Malhotra, R. 2002, AJ, 124, 2305

Nesvorný, D., Jenniskens, P., Levison, H. F., et al. 2010, ApJ, 713, 816

Nesvorný, D., Vokrouhlický, D., Bottke, W. F., \& Sykes, M. 2006, Icarus, 181, 107

Oort, J. H. 1950, Bull. Astron. Inst. Neth., 11, 91

Öpik, E. J. 1951, Proc. R. Ir. Acad. A, 54, 165

Reach, W. T., Kelley, M. S., \& Sykes, M. V. 2007, Icarus, 191, 298

Robertson, H. P. 1937, MNRAS, 97, 423

Steel, D. 1996, Space Sci. Rev., 78, 507

Steel, D. I., \& Elford, W. G. 1986, MNRAS, 218, 185

Taylor, A. D., \& Elford, W. G. 1998, Earth Planets Space, 50, 569

Wetherill, G. W. 1967, J. Geophys. Res., 72, 2429

Whipple, F. L. 1951, ApJ, 113, 464

Whipple, F. L., \& Gossner, J. L. 1949, ApJ, 109, 380

Wiegert, P., \& Tremaine, S. 1999, Icarus, 137, 84

Wiegert, P., Vaubaillon, J., \& Campbell-Brown, M. 2009, Icarus, 201, 295

Wisdom, J., \& Holman, M. 1991, AJ, 102, 1528

Wyatt, S. P., \& Whipple, F. L. 1950, ApJ, 111, 134

Younger, J. P., Reid, I. M., Vincent, R. A., Holdsworth, D. A., \& Murphy, D. J. 2009, MNRAS, 398, 350 